\title{
The genetics of tiger pelage color variations
}

\author{
Cell Research (2017) 27:954-957. doi:10.1038/cr.2017.32; published online 10 March 2017
}

\section{Dear Editor,}

The tiger (Panthera tigris) is most recognized for its dark stripes against an orange background. Less well known are three other pelage color variants: white, golden and stripeless snow white (Figure 1A). The white tiger is a polymorphism that was first seen among wild Bengal tigers (P. t. tigris) in India, with white fur and sepia brown stripes [1]. The golden tiger, also first sighted in the jungle in India, has a blonde color tone with pale golden fur and red-brown rather than black stripes [2]. The snow white tiger is almost completely white, with faint to nearly nonexistent narrow stripes on the trunk and diluted sepia brown rings on the tail [2]. During the past century, the number of wild tigers in the world has severely declined primarily due to anthropogenic reasons, and except for the wild-type orange tigers, all other variants now exist only in captivity.

The white tiger pelage is an autosomal recessive trait determined by a designated white $(W)$ locus (genotype $w /$ w) $[1,3]$; the golden color is another autosomal recessive trait coded by an independent locus termed wideband ( $W B$, referring to a wider central part of the hair shaft; genotype $w b / w b$ ) [2]; and the snow white pelage is a polygenic trait determined by dual-recessive effects at both the white and wideband loci (genotype " $w / w ; w b /$ $w b ")$ [2]. The missense mutation p.A477V in SLC45A2 has been shown to cause the background coloration switch in white tigers [4]. The only reported mammalian wideband gene thus far is the agouti signaling protein (ASIP) gene in deer mice [5], but whether the wideband gene of the golden tiger is $A S I P$, and the mechanism of interaction between the $W$ and $W B$ loci leading to the snow white pelage remain elusive. Resolving the feline wideband gene would be the ultimate step towards elucidating the genetic basis of all known coat color morphs in tigers.

Morphological examination categorizes tiger hairs into long, straight guard hairs or short, curled zigzag underhairs. Different tiger coat color variants exhibit a consistent pattern in hair composition whereby $90 \%$ of hairs are zigzag hairs, which predominantly contribute to pelage coloration. Zigzag hairs from the orange back- ground of a wild-type tiger are agouti-patterned with sub-apical pheomelanin bands and dark tips and bases, and the stripes are uniformly black (Figure 1A and Supplementary information, Figure S1A). In contrast to the wild type, zigzag hairs from a golden tiger's background fur are agouti hairs but with prolonged, lighter-colored sub-apical pheomelanin bands and dark tips/bases consistent with a "wideband" phenotype (Figure 1A-1B and Supplementary information, Figure S1A); those from the stripes are solid reddish brown. Pigmentation alteration in the golden tiger suggests a role of the $W B$ locus in extending the pheomelanin synthesis phase during the hair growth cycle. Spectrophotometric measurement showed a significant decrease in eumelanin content in the golden tiger's background zigzag hairs compared to the wildtype (Supplementary information, Figure S1B), which is consistent with the observed reduced degree of black pigmentation (Figure 1B).

As none of the tested candidate genes (MC1R, ASIP, TYR and TYRP1) showed an association with the golden tiger morph, we performed GWAS on tigers segregating at the $W B$ locus to map the wideband gene (Figure 1C). Nineteen tigers were selected from extended captive tiger pedigrees (Supplementary information, Table S1), including one golden $(W /-; w b / w b)$, eight snow white $(w / w$; $w b / w b)$ and ten white tigers $(w / w ; W B /-)$. We conducted restriction-site-associated DNA sequencing (RAD-seq) in six snow white and ten white tigers, and whole-genome sequencing (WGS) at $30 \times$ genome coverage each in one golden and two snow white tigers [4]. Combining WGS $(n=3)$ and RAD-seq $(n=16)$ data (Supplementary information, Table S1), 7015623 SNP markers were identified, of which 364553 covered at least 17 of the 19 individuals and were applied to GWAS. We detected 112 SNPs from tiger genome scaffolds 91, 97 and 1457 [6] potentially associated with the "wideband" trait $(P<$ 0.0001 ), among which 47 SNPs from scaffolds 97 and 1 457 showed the strongest association $(P=1.08 \mathrm{E}-5$, Figure 1C). Scaffolds 97 and 1457 share conserved synteny with cat chromosome B1 (Felis_catus 6.2) and represent a single continuous block with a tail-to-tail scaffold orientation. A haplotype spanning $9.30 \mathrm{Mb}$ was identified as in complete linkage disequilibrium with the $W B$ locus, 
A
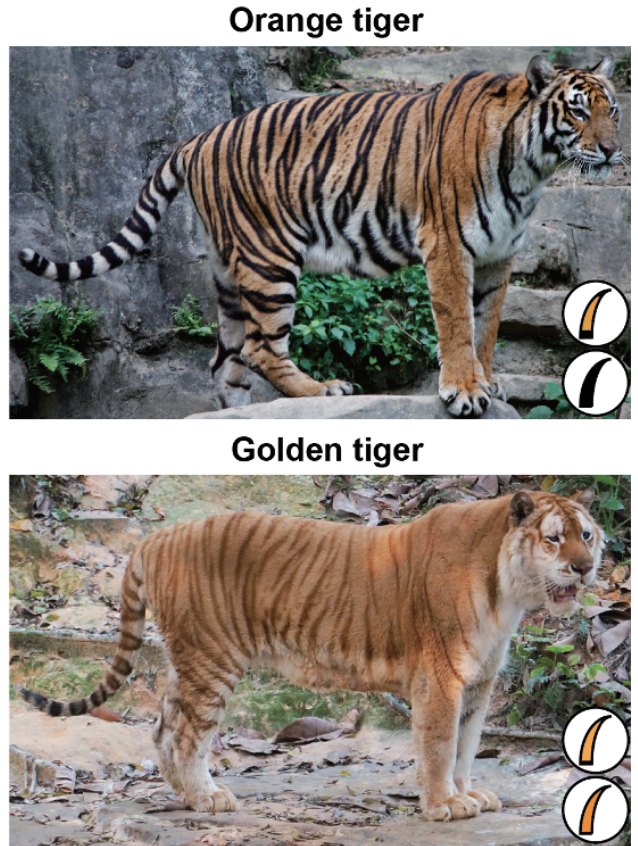

White tiger

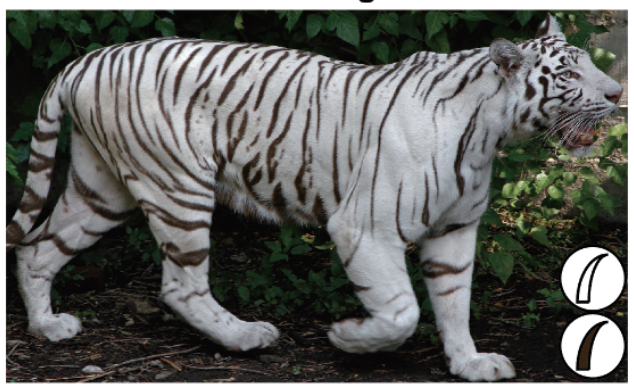

Snow white tiger

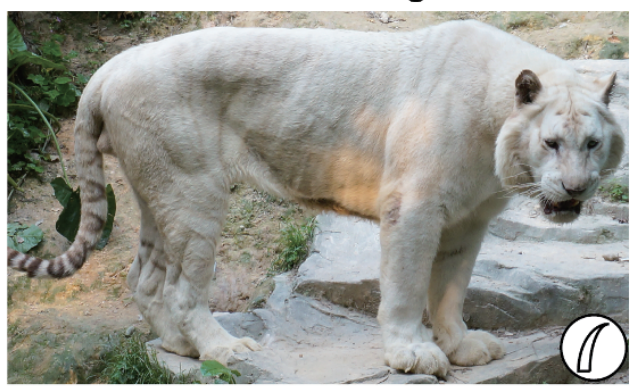

B

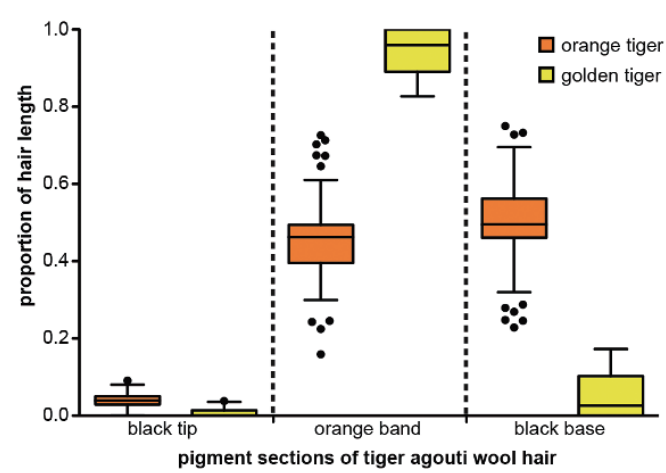

C

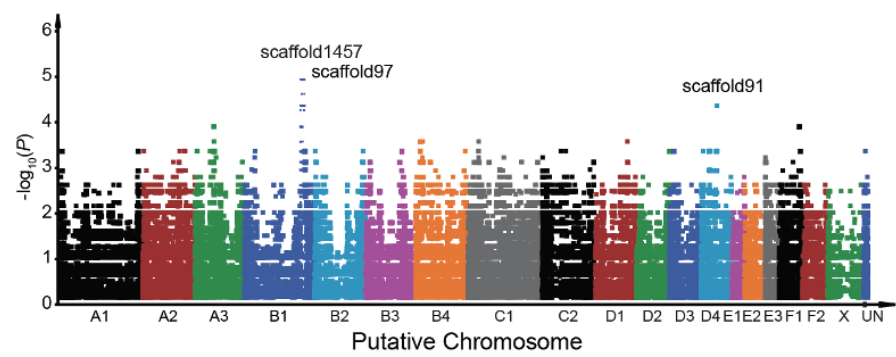

D

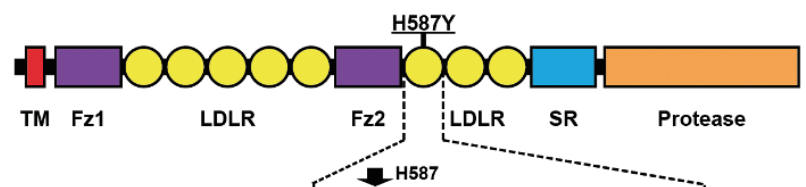

tiger(orange) ENSDNQTCLMPNEDVEECSPSHFKCGSGRCVLASRRCDGQADCDDDSDEENCG GCKERDLW

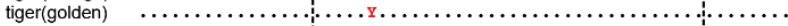

cat

dog $\quad$...E. .

panda $\quad$ Can...........

sheep $\quad$. .A...........DDE

pig $\quad \ldots \ldots \ldots \ldots$ DA.

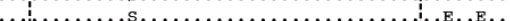

rabbit $\quad$ mons........

human $\quad$...............

gorilla
bat ................

chicken $\quad$ W.W.

E

$\leftarrow$ LDL-receptor class A domain $6 \rightarrow$

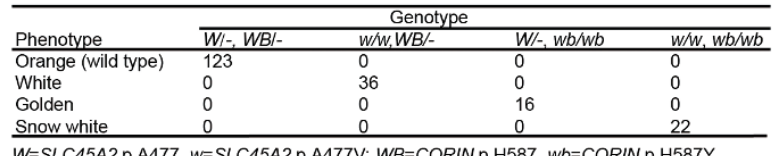

$\mathbf{F}$

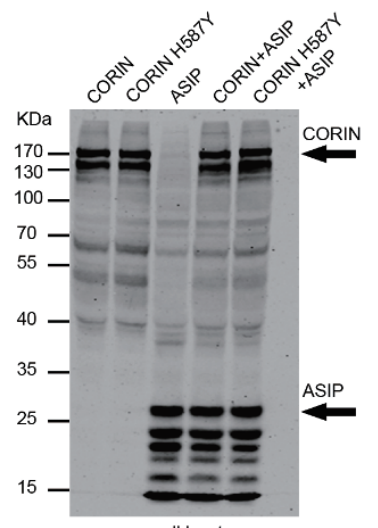

G

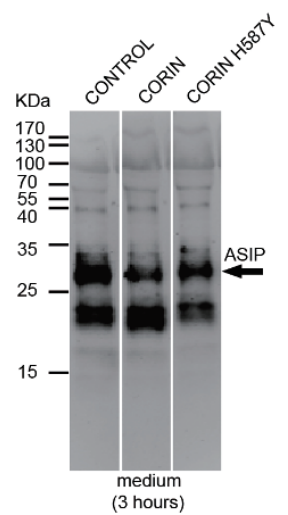


which was shared by all golden and snow white tigers (Supplementary information, Figure S1C). Scaffold 91 was excluded from further analysis as the association failed to match the recessive inheritance model of the $W B$ locus.

Gene annotation of the tiger reference genome [6] indicated 62 genes in the wideband candidate region (Supplementary information, Table S4), within which screening SNPs/indels in the coding regions identified 46 SNPs fixed in the three $w b / w b$ tiger genomes. Of the 46 SNPs, 45 showed polymorphism in the genomes of 31 additional wild-type orange tigers, representing common variations within tiger populations. Only one SNP c.1759C $>$ T (scaffold1457:2829955 C>T) identified in the coding region of CORIN is specific to golden and snow white tigers. CORIN encodes a transmembrane serine protease and is known to participate in blood pressure regulation by activating natriuretic peptides and in pigment biosynthesis by suppressing the agouti pathway [7, 8] CORIN knockout mice with agouti backgrounds exhibit prolonged agouti bands in the hair shaft [7], resembling the hair pigmentation switch in the golden tiger. CORIN c. $1759 \mathrm{C}>\mathrm{T}$ is a non-synonymous substitution resulting in a histidine-to-tyrosine substitution at amino acid residue 587 (p.H587Y). The residue H587 is located in the LDL receptor class A domain 6 of tiger CORIN, and it is evolutionarily conserved across vertebrates (Figure 1D).

A perfect correlation was detected between CORIN c. $1759 \mathrm{C}>\mathrm{T}$ and the "wideband" phenotype among unrelated individuals from wild or captive tiger populations worldwide (Figure 1E and Supplementary information, Table S1). All golden $(n=16)$ and snow white $(n=22)$ tigers were homozygous for CORIN c. $1759 \mathrm{C}>\mathrm{T}$; all orange $(n=123)$ and white $(n=36)$ tigers carried at least one copy of the wild-type allele (Figure 1E). The suppo- sition holds that CORIN c. $1759 \mathrm{C}>\mathrm{T}$ ( $\mathrm{p} . \mathrm{H} 587 \mathrm{Y})$ causes the golden tiger pelage. Genotyping analyses of CORIN p.H587Y and SLC45A2 p.A477V in the same individuals confirmed that the individual or combined effect of the two mutations corresponded to the specific tiger coat color variations (Figure 1E).

Wild-type CORIN regulates melanogenesis by suppressing the activity of ASIP, a paracrine signaling molecule that inhibits the binding of melanocortins to the transmembrane MC1R protein of melanocytes [7]. MC1R is otherwise activated by melanocyte stimulating hormone $(\alpha-\mathrm{MSH})$, leading to eumelanogenesis. The pheomelanin band in the agouti-patterned hairs is caused by a pulse of ASIP expression during hair growth, leading to a switch from eumelanin to pheomelanin production. To determine the effect of p.H587Y on CORIN's function of regulating ASIP in the tiger, we transfected HEK293 cells with tiger ASIP and/or CORIN variants. No difference was detected between the expression level of wildtype CORIN and the p.H587Y mutant in cells (Figure 1F, left panel). The secreted ASIP was reduced in the presence of wild-type CORIN, but not the p.H587Y mutant CORIN, when the culture supernatant from ASIP-expressing cells was added to cells transfected with wildtype or mutant CORIN (Figure 1G). The same result was also observed in the conditioned media of cells co-transfected with ASIP and CORIN variants (Figure 1F, right panel). The fact that secreted extracellular ASIP was degraded in the presence of wild-type CORIN suggests that CORIN functions downstream of agouti expression and likely regulates the length of the functioning period of ASIP during hair growth cycles, regardless of whether ASIP is a direct substrate of CORIN or not. The p.H587Y mutation may have impaired the function of CORIN and elongated the working period of ASIP, resulting in the

Figure 1 (A) The four coat color morphs known in tigers. Schematics of tiger zigzag hairs from the background (upper) and stripes (lower) are shown for each morph. (B) The pigmentation pattern difference in agouti zigzag hairs from orange and golden tigers. The length proportion of each component (black tip, agouti band and black base) of agouti hair is presented using Tukey's boxplots. (C) Genome-wide association plot of the tiger wideband gene. Tiger scaffolds are arranged and grouped into putative chromosomes (x axis) based on homology to the cat genome (Felis_catus 6.2). SNPs within every 500$\mathrm{Kb}$ window are plotted in a single column. Scaffolds with a high level of association $(P<0.0001)$ are marked with scaffold names. (D) Schematic diagram and partial alignment of CORIN show that H587 is located in the LDL receptor class A domain 6 and is evolutionarily conserved among vertebrates. (E) Correlation between tiger coat color phenotypes and genotypes of SLC45A2 p.A477V and CORIN p.H587Y. (F, G) Western blot analysis of lysates and medium from transfected HEK293 cells. The protein molecular weight markers are labeled on the left side of each figure. The vectors used for transfecting HEK293 cells are specified on the top of each lane. CORIN ( 170 KDa) and ASIP ( 28 KDa) with expected molecular weights are indicated by arrows. Data are representative of three independent replicas. (F) The expression level of CORIN was not affected by the p.H587Y mutation (left panel). ASIP was absent from the medium of HEK293 cells co-expressing ASIP and wild-type CORIN, but present in the medium of HEK293 cells co-expressing ASIP and CORIN p.H587Y (right panel). (G) The culture supernatants from ASIP-expressing cells were added to cells transfected with wild-type or mutant CORIN and incubated for 3 hours (left panel) and 6 hours (right panel), respectively. Secreted extracellular ASIP was degraded in the presence of wild-type CORIN, but not the CORIN p.H587Y variant. 
prolonged agouti band of the golden tiger hair.

Snow white tigers are double homozygotes for both SLC45A2 p.A477V and CORIN p.H587Y mutations, indicating that the white tiger causal mutation in conjunction with the golden tiger mutation may have diminished melanogenesis over the entire pelage. However, it must be noted that the regulation of ASIP activity by CORIN p.H587Y alone (or along with the effect of the SLC45A2 mutation) does not seem sufficient to fully explain the pigmentation change of stripes in either golden or snow white tigers. Pigmentation patterns in felids, such as stripes and spots, are maintained by an ASIP/MC1R-independent pathway involving the pattern-specific high expression of EDN3, a paracrine eumelanogenesis-stimulating hormone, and other genes such as Taqpep [9]. Further studies are thus required to fully understand the role of CORIN in interacting with other coat pattern determining genes and in modifying the pelage pigmentation.

Our study demonstrates that CORIN is a feline wideband gene. This is different from the previously reported wideband gene in deer mice, where a dominant allele of ASIP results in an enhanced level of ASIP expression [5]. Because CORIN inhibits ASIP in melanogenesis, either loss-of-function of CORIN or gain-of-function of ASIP could lead to a prolonged, "widened" agouti band in hair, demonstrating that the "wideband" phenotype in mammals has multiple origins.

Through WGS, we identified the genetic basis of golden tigers as a non-synonymous mutation p.H587Y of CORIN; together with the white tiger mutation SLC45A2 p.A477V, the combined effect of these two mutations leads to the nearly complete loss of stripes and pigmentation in the snow white tiger. Therefore, the molecular basis of all four major pelage variants observed in tigers, first originating from the wild as natural polymorphism and later maintained by captive breeding, was revealed. While the white tiger morph is considered a natural genetic polymorphism without compromising fitness or survival in the wild, the physiological effects of the CORIN variant on the golden and snow white tigers remain unclear, as CORIN has functions in multiple pathways or processes, including blood pressure regulation and trophoblast invasion. In the wild, confirmed records of golden tigers are extremely rare and much less common than those of the white tiger in centuries past. Studies of non-model species will continue to reveal novel genetic variants with large phenotypic effects, and this is crucial to ultimately elucidating the genetic foundation of the diverse traits of life.

\section{Accession numbers}

RAD-seq reads have been deposited in the NCBI BioProject database under the accession number PRJNA290531 and Sequence Read Archive (SRA) under the accession number SRP061401. The cat cDNA sequence has been deposited in the NCBI GenBank under the accession number KT750959.

\section{Acknowledgments}

We thank all the collaborators, zoos and private owners for providing specimens, particularly SJ O'Brien, M Menotti-Raymond, V David, R Hanson, "Doc" B Antle, YS Zhong, RA Qiu, BH Li, ZQ Lei, WH Yang, J Dong, and JQ Zheng. We thank L Mhuulu, HR Xue, YC Liu, HH Feng, H Yu, L Miao, C Xie, R Guo, Q Li, JX Feng, DY Xu and RZ Yang for technical assistance and GS Barsh for helpful comments. This research was supported by the Peking-Tsinghua Center for Life Sciences and the National Natural Science Foundation of China (31271320, 31471179, 31200919).

Xiao Xu ${ }^{1}$, Gui-Xin Dong ${ }^{2}$, Anne Schmidt-Küntzel ${ }^{3}$, Xue-Li Zhang, ${ }^{2}$, Yan Zhuang ${ }^{1}$, Run Fang ${ }^{1}$, Xin Sun ${ }^{1}$, Xue-Song $\mathrm{Hu}^{1}$, Tian-You Zhang ${ }^{2}$, Han-Dong Yang ${ }^{2}$, De-Lu Zhang ${ }^{2}$, Laurie Marker ${ }^{3}$, Zheng-Fan Jiang ${ }^{1}$, Ruiqiang $\mathrm{Li}^{4}$, Shu-Jin Luo ${ }^{1}$

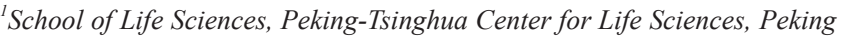
University, Beijing 100871, China; ${ }^{2}$ Chimelong Safari Park, Chimelong Group Co., Panyu, Guangzhou 511430, China; ${ }^{3}$ Life Technologies Conservation Genetics Laboratory, Cheetah Conservation Fund, Otjiwarongo 9000, Namibia; ${ }^{4}$ Novogene Bioinformatics Institute, Beijing 100083, China Correspondence: Shu-Jin Luo

E-mail: 1uo.shujin@pku.edu.cn

\section{References}

1 Thornton IW, Yeung K, Sankhala K. J Zool 1967; 152:127-135.

2 Zebley M, Olson T. The genetic and biochemical basis for the "golden tabby" and "snow white" Bengal tigers. Annual Proceedings-American Association of Zoo Veterinarians: Hill's Division, Riviana Foods 1997:78.

3 Robinson R. Genetica 1990; 46:1-31.

4 Xu X, Dong GX, Hu XS, et al. Curr Biol 2013; 23:1031-1035.

5 Linnen GR, Kingsley EP, Jensen JD, et al. Science 2009; 325:10951098.

6 Cho YS, Hu L, Hou H, et al. Nat Commun 2013; 4:2433.

7 Enshell-Seijffers D, Lindon C, Morgan BA. Development 2008; 135:217-225.

8 Yan W, Sheng N, Seto M, et al. J Biol Chem 1999; 274:14926-14935.

9 Kaelin CB, Xu X, Hong LZ, et al. Science 2012; 337:1536-1541.

(Supplementary information is linked to the online version of the paper on the Cell Research website.) 